\title{
Stacking Faults in Layered Electrode Materials: Developments in Structure Solutions for Diffraction Data
}

Alexander Brady, Weiwei Sun, Jessica L. Durham, Esther S. Takeuchi, Kenneth J. Takeuchi, Amy C. Marschilok, Michael Naguib, Lukas Vlcek, Hsiu-Wen Wang

Email Contact: bradyab@ornl.gov

Various layered transition metal oxides are attractive electrode materials in battery or supercapacitor applications [1]. However, many layered materials have stacking faults. While it is sometimes possible to prevent or remove these stacking faults during synthesis, there is some evidence that stacking faults may improve electrochemical performance [2]. Stacking faults present a unique challenge for structural analysis, the stacking randomness leads to complex diffraction patterns dominated by asymmetric peak profiles. The ability to extract structural models from experimental data with the atomic-level detail needed to guide and validate leading nanoscale theories is a unifying challenge in the study of materials chemistry. In this talk, two study cases are presented. In the first example, we demonstrate how the direct modelling of stacking fault structures can improve estimates of lattice parameters and crystallite size in the silver ferrite cathode (Figure 1A) [3]. In the second example, we present multilevel modelling approaches combined with molecular dynamics simulations to fully characterize the structure and interlayer correlations in 2-dimentionally stacked titanium carbonitride, $\mathrm{Ti}_{3} \mathrm{CN} T_{x}$ MXenes ( $T=$ terminal $-\mathrm{F}$ and $-\mathrm{O}$ atoms) (Figure 1B) [4].
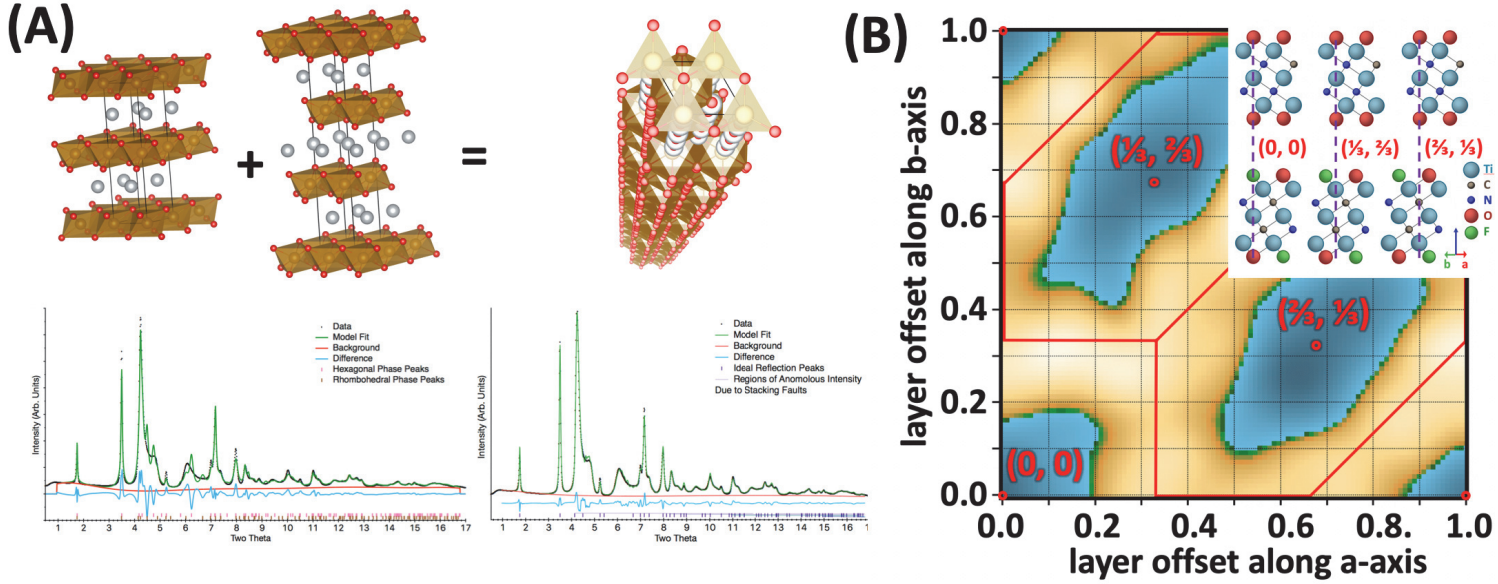

Figure 1. (A) Silver Ferrite stacking fault modelling (B) Interlayer correlations in $\mathrm{Ti}_{3} \mathrm{CN}$ MXenes.

1. Whittingham, M. Stanley. "Lithium batteries and cathode materials." Chemical reviews 104.10 (2004): 4271-4302.

2. Kim, Jung-Min, and Hoon-Taek Chung. "Electrochemical characteristics of orthorhombic LiMnO2 with different degrees of stacking faults." Journal of power sources 115.1 (2003): 125-130.

3. Durham, Jessica L., et al. "Electrochemical (de) lithiation of silver ferrite and composites: mechanistic insights from ex situ, in situ, and operando X-ray techniques." Physical Chemistry Chemical Physics 19.33 (2017): 22329-22343.

4. Sun, Weiwei, et al. "Atomic-scale characterization and modeling of Ti3CN: structure change and elastic reponse." In Preparation. 